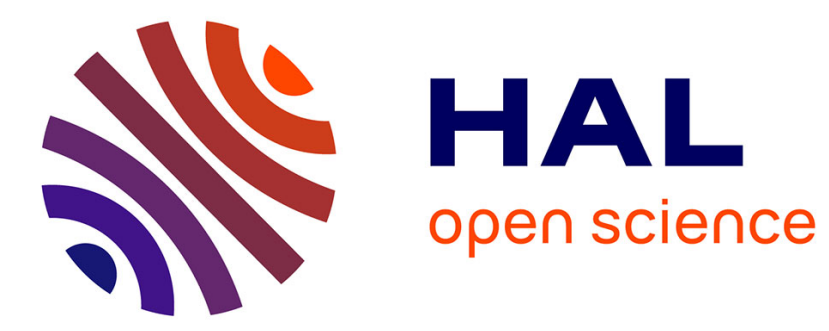

\title{
On fractal nature of groundwater level fluctuations due to rainfall process
}

Maminirina Joelson, Jacques Golder, Philippe Beltrame, Marie-Christine

Neel, Liliana Di Pietro

\section{- To cite this version:}

Maminirina Joelson, Jacques Golder, Philippe Beltrame, Marie-Christine Neel, Liliana Di Pietro. On fractal nature of groundwater level fluctuations due to rainfall process. Chaos, Solitons \& Fractals, 2016, 82, pp.103-115. 10.1016/j.chaos.2015.11.010 . hal-01277631

\section{HAL Id: hal-01277631 \\ https://hal.science/hal-01277631}

Submitted on 22 Feb 2016

HAL is a multi-disciplinary open access archive for the deposit and dissemination of scientific research documents, whether they are published or not. The documents may come from teaching and research institutions in France or abroad, or from public or private research centers.
L'archive ouverte pluridisciplinaire HAL, est destinée au dépôt et à la diffusion de documents scientifiques de niveau recherche, publiés ou non, émanant des établissements d'enseignement et de recherche français ou étrangers, des laboratoires publics ou privés. 
See discussions, stats, and author profiles for this publication at:

https://www.researchgate.net/publication/285588327

\section{On fractal nature of groundwater level fluctuations due to rainfall process}

ARTICLE · JANUARY 2016

READS

14

5 AUTHORS, INCLUDING:

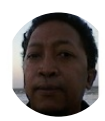

Joelson Maminirina

Université d'Avignon et des Pays du V...

Philippe Beltrame

53 PUBLICATIONS 120 CITATIONS

Université d'Avignon et des Pays du V..

25 PUBLICATIONS 92 CITATIONS

SEE PROFILE

SEE PROFILE 


\title{
On fractal nature of groundwater level fluctuations due to rainfall process
}

\author{
Maminirina Joelson $^{\mathrm{a}, \mathrm{b}, *}$, Jacques Golder ${ }^{\mathrm{a}, \mathrm{b}}$, Philippe Beltrame ${ }^{\mathrm{a}, \mathrm{b}}$, \\ Marie-Christine Néel ${ }^{\mathrm{a}, \mathrm{b}}$, Liliana Di Pietro ${ }^{\mathrm{b}}$ \\ a Department of Physics,University of Avignon, 74 rue Louis Pasteur, 84029 Avignon cedex 1, France \\ b UMR EMMAH, INRA Domaine St Paul, Site Agroparc, 84914 Avignon cedex 9, France
}

\section{A R T I C L E I N F O}

\section{Article history:}

Received 12 March 2015

Accepted 9 November 2015

\section{Keywords:}

Groundwater level fluctuations

Fractal

Scale invariant

High order spectra analysis

Lévy stable distribution

Hurst exponent

\begin{abstract}
A B S T R A C T
Hourly resolution time series of groundwater level fluctuations are analyzed after removing the seasonal cycle. It is found that fluctuations of groundwater levels have fractal scaling and a persistent behavior. We show also that groundwater level fluctuations exhibit non-Gaussian heavy tailed probability distribution that is well fitted by the Lévy stable distribution. Implications of the present results on the groundwater system modeling as a fractional Lévy motion and the connection with the anomalous diffusion inside the soil are discussed.
\end{abstract}

(c) 2015 Elsevier Ltd. All rights reserved.

\section{Introduction}

The distribution of groundwater and dynamic fluctuations in groundwater levels have direct impacts on the environment quality. This is particularly the case of the problems raised by drinking water supplies and subsurface water quality. Level fluctuations of groundwater system are dynamic responses of the system to its recharge and discharge. The groundwater system may be considered as a complex dynamic system characterized by non-stationary input (recharge), output (base flow), and response (groundwater levels) (see e.g. [4,22]). The recharge of groundwater system is mainly ensured by the rainfall process that pass through the soil layer. This infiltration process is known to transport contaminants on the soil surface toward the groundwater system. Various works were conducted to study impacts of rainfall process on groundwater dynamic. These

\footnotetext{
* Corresponding author at: Department of Physics,University of Avignon, 74 rue Louis Pasteur 84029 Avignon cedex 1, France. Tel.: +33 624234515. E-mail address: joelson.maminirina@avignon.inra.fr (M. Joelson).
}

works are mostly concerned by large scale evolution and combine generally modeling and data mining approaches $[6,8,9,36,41]$. The present study deals with the groundwater dynamic at a smaller scale related to the area of a plot. The study is based on experiments conducted on the experimental field of the French Institute for Agricultural Research at the INRA-Avignon Research Center. This site is devoted to analyze the mass transport process under the soil surface. The present paper aims to analyze the temporal characteristics of groundwater fluctuations in a region characterized by rapid intense rainfall and violent storm. The goal of the work is to show that under conditions described above the statistical behavior of the groundwater level fluctuations due to rainfall events is non-Gaussian and found to be well represented by the class of stable heavy tailed probability distribution. Scaling properties of the observed groundwater level fluctuations are quantified. The obtained results suggest that the dynamic of the groundwater level fluctuations has persistent behavior and a fractal property revealing a long memory effect and a probably complex relation with the rainfall process. Also, our results do not support the use the Brownian and the fractional Brownian motions to describe the dynamic 
- Piezometers position

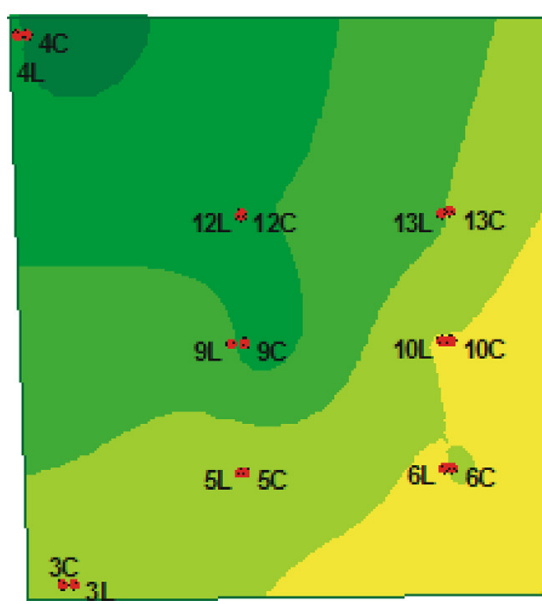

Fig. 1. Relief map and piezometers position. The three measure points are located in points 1,2 and 3 (in the corners of the map). of the groundwater level fluctuations $[46,48]$. According to these findings, implications on the groundwater dynamics and the characterization of the infiltration process through the soil layer as a random porous media are discussed. The paper is presented in five parts. The Section 2 is devoted to the presentation of the experiment. In Section 3 we present the analysis methods applied in this paper and their mathematical properties. Section 4 is focused on the presentation and the interpretation of the results obtained from the data experiments. We end the paper with a discussion in Section 5 about the implication and the contribution of the present paper on the study of groundwater dynamics.

\section{Field measurements}

The investigations were carried out from data measured from the workshop site "Fontanille" of the Research Center of INRA Avignon. The site is located in an extensive, almost flat area, about $8 \mathrm{~km}$ South-Est of Avignon $\left(43^{\circ} 54^{\prime} 58^{\prime \prime} \mathrm{N}, 4^{\circ} 52^{\prime} 58^{\prime \prime} \mathrm{E}\right)$ in the Vaucluse region which is situated in South-Est of France at approximately $100 \mathrm{~km}$ North of the Mediterranean facade. The surface under study is $0.64 \mathrm{Ha}$. A general view of the experiment field and the relief map are given in Fig. 1. The ground surface altitude varies slightly around $31 \mathrm{~m}$. A more detailed description of the workshop site and its technical characteristics are available in [7].

The Vaucluse region is subjected to a Mediterranean climate which is characterized by a summer drought, a strong sunshine and an irregular rainfall. The mean annual temperature is $14.8^{\circ} \mathrm{C}$. However, this value does not represent the strong variation between the mean annual temperature in winter $\left(6.5^{\circ} \mathrm{C}\right)$ and in summer $\left(22.6^{\circ} \mathrm{C}\right)$ seasons. The mean annual precipitation is $650-700 \mathrm{~mm} / \mathrm{a}$ which is formed by 80 100 of rainy days mainly during April-May and SeptemberOctober. As a general remark of the climate, rainy days are rare but they often induce intense rainfall and violent storm.

The groundwater system is part of the recent quaternary aquifer of the Lower Durance and is mainly composed of an
Table 1

Statistics from the groundwater height fluctuations.

\begin{tabular}{lll}
\hline Piezometers & Mean value $(\mathrm{m})$ & Std $(\mathrm{m})$ \\
\hline 1C & 26.552 & 0.258 \\
2L & 26.625 & 0.265 \\
3C & 26.256 & 0.244 \\
\hline
\end{tabular}

alluvial plain. We are in presence of a free layer of quaternary sands ranging between 4 and $6 \mathrm{~m}$ below the soil surface.

As said above, the site is devoted to the study of mass transfer process from soil surface to groundwater and is equipped with various instruments intended to measure the evolution of different quantities involved in the physical phenomena under consideration. In particular for the present study, we focus on fluctuations of the groundwater level due to the rainfall. The general way to quantify these fluctuations requires the use a piezometer network. Each piezometer is made of a borehole in which pressure probe is put and designed for a measure at a single point within the aquifer.

The location of the piezometer's network is given in Fig. 1. The network is located in measure points numbered from 1 to 14 . We focus here on the first three piezometers located at points 1,2 and 3 that allow us to study time characteristics of the level fluctuations and also to estimate an eventual space variability linked to an eventual soil heterogeneity. For each location, time series data of 10.000 measurements are available corresponding to 14 months record duration starting on February 02th 2005 and ending on March 27th 2006. A brief statistical summary of the data is given in Table 1 . Of interest is that values of groundwater level are given here following the NGF (Nivellement Général de la France) norm that takes the sea level as a reference point (It corresponds to the meter ASL (Above Sea Level) system.) .

As a general remark from Table 1, the three time series present very similar statistics. This means that no significant space variability is found between these three positions. This interpretation was confirmed by more advanced analysis. The three time series was processed in the same man- 


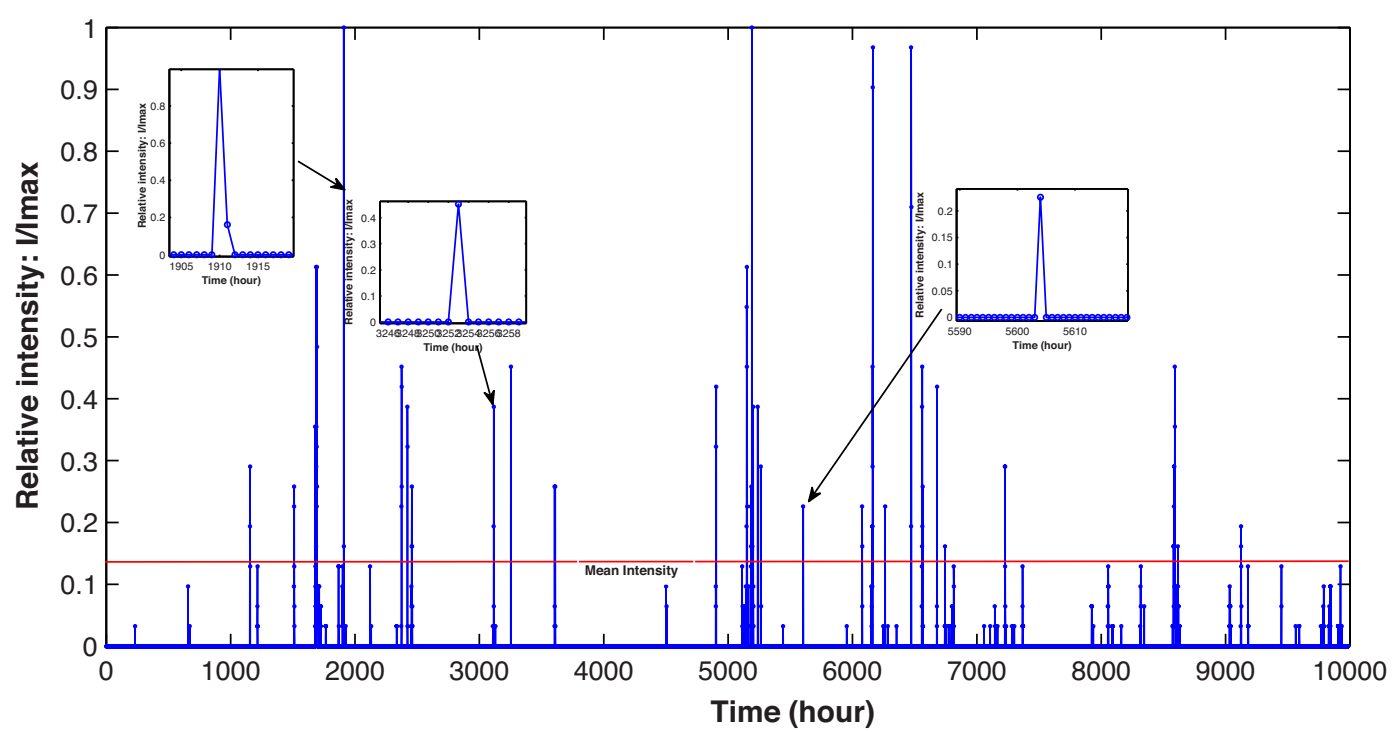

Fig. 2. Rainfall relative intensity showing some examples of short duration event with strong intensity (upper than two times the mean intensity).

Table 2

Statistics of rainfall events from the time series.

\begin{tabular}{lrr}
\hline Event duration & Number & Proportion (\%) \\
\hline $1-3 \mathrm{~h}$ & 92 & 79.31 \\
$4-6 \mathrm{~h}$ & 18 & 15.52 \\
$7 \mathrm{~h}$ and more & 6 & 5.17 \\
\hline
\end{tabular}

ner for each analysis methods proposed in the present study. Owing to this similarity between the three cases, we will present only the results relating to the time series from measure point 1 (hereafter named as $\eta(t)$ ) in this paper.

Simultaneously with the groundwater level measurement, a tipping-bucket rain gauge was used to collect rainfall data with a time resolution of $1 \mathrm{~h}$ and an intensity resolution less than $3 \%$ at a rainfall intensity of order $50 \mathrm{~mm} / \mathrm{h}$. More detailed description and extended analysis about the precision and the accuracy of the installed rainfall measurement system can be found in the technical notes [19]. Also, rainfall time series is made of 10000 points. Table 3 gives a brief summary of the rainfall duration distribution. Table 2 shows that around $80 \%$ of rainfall events last less than $3 \mathrm{~h}$ but they can have rapidly a strong intensity (see examples in Fig. 2).

\section{Analysis methods}

In this section we present briefly different analysis methods that were applied to the data. In addition to the classical Fourier spectral methods, technique of simulation of surrogate data and model of probability density function for nonGaussian stable distribution will be used in this paper. The aims of these methods are respectively to identify the correlated with rainfall events component of the groundwater level variation, to characterize its scale properties and to fit its probability distribution.

\subsection{Fourier transform of time series}

The first step in our analysis will be to use Fourier transform that allows one to represent the time series as a sum of combinations of amplitudes and phases as:

$\hat{\eta}(f)=\int_{-\infty}^{\infty} \eta(t) e^{-2 \pi i t f} d t$

where $\eta(t)$ is the initial time series and $\hat{\eta}(f)$ is its Fourier transform. Eq. (1) is the decomposition of the time series in term of harmonic component. Real data from experiments being of discrete nature

$\left\{\eta\left(t_{1}\right), \eta\left(t_{2}\right), \ldots, \eta\left(t_{i}\right), \ldots, \eta\left(t_{n}\right)\right\}$

then it is convenient to replace the integral form of (1) with the corresponding discrete form as:

$\hat{\eta}\left(f_{k}\right)=\sum_{j} \eta\left(t_{j}\right) e^{-2 \pi i t_{j} f_{k}} \delta t$

We note that in Eq. (2), $\delta t$ is the time step of the data record and $t_{i}=i \cdot \delta t$ is the discrete time. In the Fourier space, the frequency values are given by $f_{k}=k \cdot \delta f$ where $\delta f=1 / \delta t$ defines the frequency resolution. In general, $\hat{\eta}\left(f_{k}\right)$ will be complex of the form $a\left(f_{k}\right)+i b\left(f_{k}\right)$. Of interest is that $\hat{\eta}\left(f_{k}\right)$ may be expressed in polar form though:

$\hat{\eta}\left(f_{k}\right)=\left|\hat{\eta}\left(f_{k}\right)\right| e^{i \Phi\left(f_{k}\right)}$

where $\left|\hat{\eta}\left(f_{k}\right)\right|=\sqrt{a^{2}+b^{2}}$ is the Fourier amplitude at frequency $f_{k}$ and $\Phi\left(f_{k}\right)=\tan ^{-1}\left(\frac{b}{a}\right)$ represents the phase. Also, one can define the autocorrelation function as (see for example in Koopmans [17] p.30):

$C_{\eta}(\tau)=\lim _{T \rightarrow+\infty} \frac{1}{2 T} \int_{-T}^{T} \eta(t+\tau) \eta(t) d t$

Eq. (4) quantifies the coherency or the similarity level between observations as a function of the time separation $\tau$ between them. The Wiener Khinchin theorem [17] states 
that the autocorrelation function $C_{\eta}(\tau)$ is the inverse Fourier transform of the power spectral density.

$S_{\eta}(f)=\int_{-\infty}^{\infty} C_{\eta}(\tau) e^{-i 2 \pi f \tau} d \tau$

The spectral density (5) gives the frequency distribution of the energy of $\eta(t)$. It constitutes the second statistical moment in Fourier frequency domain. Again, discrete form of the spectrum in (5) reads as:

$S_{\eta}\left(f_{k}\right)=\sum_{j} C_{\eta}\left(\tau_{j}\right) e^{-i 2 \pi \tau_{j} f_{k}}$

From (3), it is straightforward that $S_{\eta}(f)=\left|\hat{\eta}\left(f_{k}\right)\right|^{2}$. From this later expression, one can remark that the phase information is discarded. This means the limit of the spectral analysis. Indeed, although the spectrum function gives the frequency distribution of the energy, it does not provide any explanation on the origin of this later. Phase information can be assessed with higher order spectral analysis in term of nonlinear interactions between Fourier components. Higher order spectral analysis is an useful tool to highlight deterministic chaotic dynamics.

\subsection{Testing coherency with rainfall signal by the surrogate data method}

Let us design by $\lambda(t)$ the time series of the rainfall. The main idea of this section is to analyze the coherency between both time series $\lambda(t)$ and $\eta(t)$. For such purpose, we first define the cross correlation function by generalizing the relation (4) as:

$C_{\lambda \eta}(\tau)=\lim _{T \rightarrow+\infty} \frac{1}{2 T} \int_{-T}^{T} \lambda(t+\tau) \eta(t) d t$

By applying the Fourier transform to (7), it may be shown that the cross correlation $C_{\lambda \eta}(\tau)$ depends on the phase shift between the time series $\lambda(t)$ and $\eta(t)$ and their Fourier amplitudes. In Fourier space, it is more convenient to define the cross correlation between both time series by a normalized coherency bounded by 0 and 1 and phase function as:

$R_{\lambda \eta}(f)=\frac{\left|S_{\lambda \eta}(f)\right|^{2}}{S_{\lambda}(f) S_{\eta}(f)}$

$P h_{\lambda \eta}(f)=\arctan \left(\frac{-\operatorname{Im}\left(S_{\lambda \eta}(f)\right)}{\operatorname{Re}\left(S_{\lambda \eta}(f)\right)}\right)$

where $S_{\lambda \eta}(f)$ is the Fourier transform of $C_{\lambda \eta}(\tau)$ and $S_{\eta}(f)$ and $S_{\lambda}(f)$ are respectively the Fourier transform of the autocorrelation of each time series according to relation (5).

The idea that a part of the groundwater level signal $\eta(t)$ is highly correlated with the rainfall time series and then constitutes the rainfall-response of groundwater level is formulated as a null hypothesis. If this hypothesis is significant then appropriate amplitude and phase relationships have to exist in the groundwater signal. One way to test the null hypothesis is then to construct from the groundwater signal $\eta(t)$ a surrogate data that does not contain any phase properties. Starting from the Fourier transform $\hat{\eta}(f)$, the surrogate data is made by replacing the phase function $\Phi(f)$ in the expression (3) with a random uniform values and taking after the inverse Fourier transform to build a new time series.
The surrogate method destroys the phase properties but preserves other properties such as spectrum or probability distribution [43]. In order to validate the significance of the null hypothesis we compare observed correlations of rainfall time series with real and surrogate data.

\subsection{Higher order spectral analysis}

The first tool for nonlinear spectral analysis comes from the bispectrum that is the Fourier transform of the triple correlation [27,31]. It reads as:

$R(u, v)=\hat{\eta}(u) \hat{\eta}(v) \hat{\eta}(-u-v)$

$R$ is a function of two frequency variables $u$ and $v$ and its inverse Fourier transform writes as

$$
\begin{aligned}
r(\tau, \theta)= & \frac{1}{4 \pi^{2}} \int_{-\infty}^{+\infty} \int_{-\infty}^{+\infty} \hat{\eta}(u) \hat{\eta}(v) \hat{\eta}(-u-v) \\
& \times e^{i(u \tau+v \theta)} d u d v
\end{aligned}
$$

Recalling that the inverse Fourier transform of the product of two functions is given by their convolution in time space; it is straightforward to show that $r(\tau, \theta)$ is of the form:

$r(\tau, \theta)=\int_{-\infty}^{+\infty} \eta(t) \eta(t+\tau) \eta(t+\theta) d t$

The expression (12) generalizes the autocorrelation function and allows us to describe higher statistical properties of the time series [31]. In particular, let usrecall that Gaussian distribution is fully defined by the two first moments: mean and variance. Then a Gaussian process is also entirely described by its autocorrelation function and does not possess higher order correlation functions. Thus, the bispectrum is an indicator of the non-Gaussianity property of the time series [11]. The bispectrum may be seen also as a quantification of the interactions strength between three Fourier components. Three components $(u, v, u+v)$ interactions can be ascribed to nonlinearities of the quadratic type. These later are characteristic of low dimensional chaotic dynamics [15]. In practice, it is more convenient to use the bicoherence (normalized form of the bispectra) giving a bounded quantity between 0 and 1 .

\subsection{Non-Gaussian probability distribution}

In order to give insight on the probability distribution of the ground water fluctuations level, we will use the stable laws called also $\alpha$-stable law. The stable laws were introduced by Lévy in [21] during his investigations of the behavior of the sums of independent identically distributed random variables. Briefly, we recall here some basic properties of the Lévy $\alpha$-stable law. A stable distribution law is determined by four parameters: an index of stability $\alpha$, a location parameter $\mu$, a skewness parameter $\beta$ and a scale parameter $\gamma$. A random variable $\mathrm{X}$ is said to have a stable distribution (noted as $\mathrm{X} \sim S_{\alpha}(\beta, \gamma, \mu)$ ) if its characteristic function satisfies

$\Phi_{X}(t)=\exp \left(i \mu t-\gamma^{\alpha}|t|^{\alpha}[1-i \beta \operatorname{sign}(t) \mathrm{W}(\alpha, t)]\right)$

where 
$\mathrm{W}(\alpha, t)=\left\{\begin{array}{cl}\tan \left(\frac{\pi \alpha}{2}\right) & \text { if } \alpha \neq 1 \\ -\frac{2}{\pi} \log |t| & \text { if } \alpha=1\end{array}\right.$

and

$\operatorname{sign}(t)= \begin{cases}1 & \text { if } t>0 \\ 0 & \text { if } t=0 \\ -1 & \text { if } t<0\end{cases}$

The stability index also called as the characteristic exponent or the tail index satisfies $0<\alpha \leq 2$ and the skewness parameter $-1 \leq \beta \leq 1$. The location parameter $\mu$ is defined only when the stability index is $\alpha>1(\mu \in \mathbf{R})$. In this case, the first absolute moment is given by $\left\langle|X|>=\frac{2 \gamma}{\pi} \Gamma(1-1 / \alpha)\right.$ where $\Gamma$ (.) is the gamma function. If $\beta=0$, then the distribution is symmetric around $\mu$. The scale parameter also called as dispersion parameter has a real positive value $\gamma>0$ that determines the width of the distribution law. The location parameter $\mu$ describes the shift of the peak. The tail index $\alpha$ determines the rate at which the tails of the distribution taper off.

An important property of $\alpha$-stable distributions is the role that they play in the generalized central limit theorem. The generalization of the central limit theorem due to Gnedenko and Kolmogorov [10] states that the sum of a number of random variables with a power-law tail (Paretian tail) distributions will tend to an $\alpha$-stable distribution. More precisely, using the generalized central limit theorem, it can be shown that:

$\left\{\begin{array}{l}\lim _{t \rightarrow \infty} t^{\alpha} \operatorname{Pr}(X>t)=C_{\alpha}(1+\beta) \gamma^{\alpha} \\ \lim _{t \rightarrow \infty} t^{\alpha} \operatorname{Pr}(X<-t)=C_{\alpha}(1-\beta) \gamma^{\alpha}\end{array}\right.$

where $C_{\alpha}=\frac{1}{\pi} \Gamma(\alpha) \sin \left(\frac{\pi \alpha}{2}\right)$. From (14), it yields that in general, the $p$ th order moment of a stable random variable is finite if and if only $p<\alpha$. We note also from (14) that $\alpha$ stable distribution laws are well adapted to model self-affine behavior. Indeed, the power-law tail behavior in (14) is directly linked with a possibly fractal dynamic in the physical process [39].

To perform the probability distribution of the Lévy $\alpha$ stable random variable $X$, we adopt the integral expression given by Zolotarev in [49].

The main interest of Zolotarev's formulas is that instead of others integral expression, they do not include infinite integral and then are well adapted to numerical computations. In which follow, we give Zolotarev's formulas of the PDF of a stable random variable $X \sim S_{\alpha}(\beta, \gamma, \mu)$. First, we consider the case of a random variable $\mathrm{X}_{0} \sim \mathrm{S}_{\alpha}(\beta, 1,0)$. By setting $\zeta=-\beta \tan \frac{\pi \alpha}{2}$, the PDF of $X_{0}$ can be expressed as:

if $\alpha \neq 1$

$$
f_{\alpha, \beta, 1,0}(x)= \begin{cases}\frac{\alpha(x-\zeta)^{\frac{1}{\alpha-1}}}{\pi|\alpha-1|} \int_{-\zeta}^{\frac{\pi}{2}} \mathrm{~V}(\theta, \alpha, \beta) & \\ \times \exp \left\{-(x-\zeta)^{\frac{\alpha}{\alpha-1}}\right. & \\ \times \mathrm{V}(\theta, \alpha, \beta)\} d \theta, & \text { if } x>\zeta \\ \frac{\Gamma\left(1+\frac{1}{\alpha}\right) \cos (\xi)}{\pi}, & \text { if } x=\zeta \\ f_{\alpha,-\beta, 1,0}(-x), & \text { if } x<\zeta\end{cases}
$$

$$
\begin{gathered}
\text { if } \alpha=1 \\
f_{1, \beta, 1,0}(x)= \begin{cases}\frac{1}{2|\beta|} \exp \left(-\frac{\pi x}{2 \beta}\right) \int_{-\frac{\pi}{2}}^{\frac{\pi}{2}} \mathrm{~V}(\theta, 1, \beta) & \\
\times \exp \left(-\exp \left(-\frac{\pi x}{2 \beta}\right)\right. & \text { if } \beta \neq 0 \\
\times \mathrm{V}(\theta, 1, \beta)) d \theta, & \text { if } \beta=0 \\
\frac{1}{\pi\left(1+x^{2}\right)}, & \text { i } 3\end{cases}
\end{gathered}
$$

where $\xi=\frac{1}{\alpha} \arctan (-\zeta)$ and

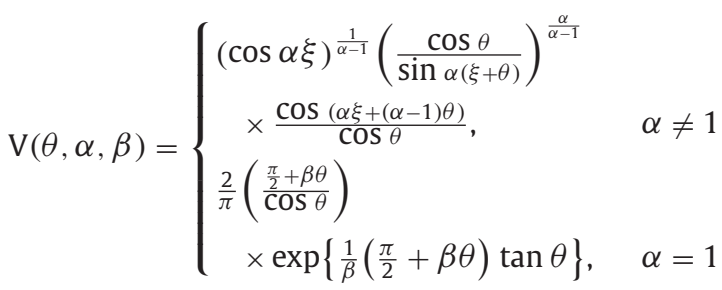

From the probability distribution function given by (15), one can easily build the probability distribution function of the random variable $\mathrm{X}$ with the help of the stability property, $X=\gamma X_{0}+\mu \sim S_{\alpha}(\beta, \gamma, \mu)$.

To fit the four stable parameters from experiment data, we will use the sample characteristic function method (see for example in $[16,18])$. The method is based on regression type on the log-characteristic function [14]. Indeed, the logarithm of the real part and the imaginary part of the logcharacteristic function are linear and then, give rise to a regression model with the data.

\section{Data analysis and results}

In this section, we present the results obtained by applying to our data series the analysis methods developed in the previous section.

\subsection{Detecting rainfall correlated component of groundwater level fluctuations}

The first step of the paper is to detect and extract the rainfall correlated component of the groundwater level variation. As a preliminary investigation, the graphs of the two time series $\lambda(t)$ and $\eta(t)$ are shown in Fig. 3 in a synchronized time. The upper part of the figure depicts the groundwater level variation when the bottom part represents the time evolution of the rainfall. A qualitative inspection from the groundwater level variation allows one to remark that the time series $\eta(t)$ is formed by two distinct components characterized by a large and a small time scales. According to the characteristic time scale of the rainfall time series, it is 'a priori' clear that the large scale component of the groundwater level corresponds more to the seasonal variation than a response of this later to the rainfall forcing. Owing to the intensive practice of the gravity flow irrigation in the region during second half of the year, one can also note the reversed sense of the large time scale variation of the groundwater level compared with the natural annual evolution.

For the sake of completeness, we perform this qualitative interpretation by computing the correlation between both time series $\lambda(t)$ and $\eta(t)$. The results in term of coherency and phase shift functions are shown in Fig. 4 according to the 

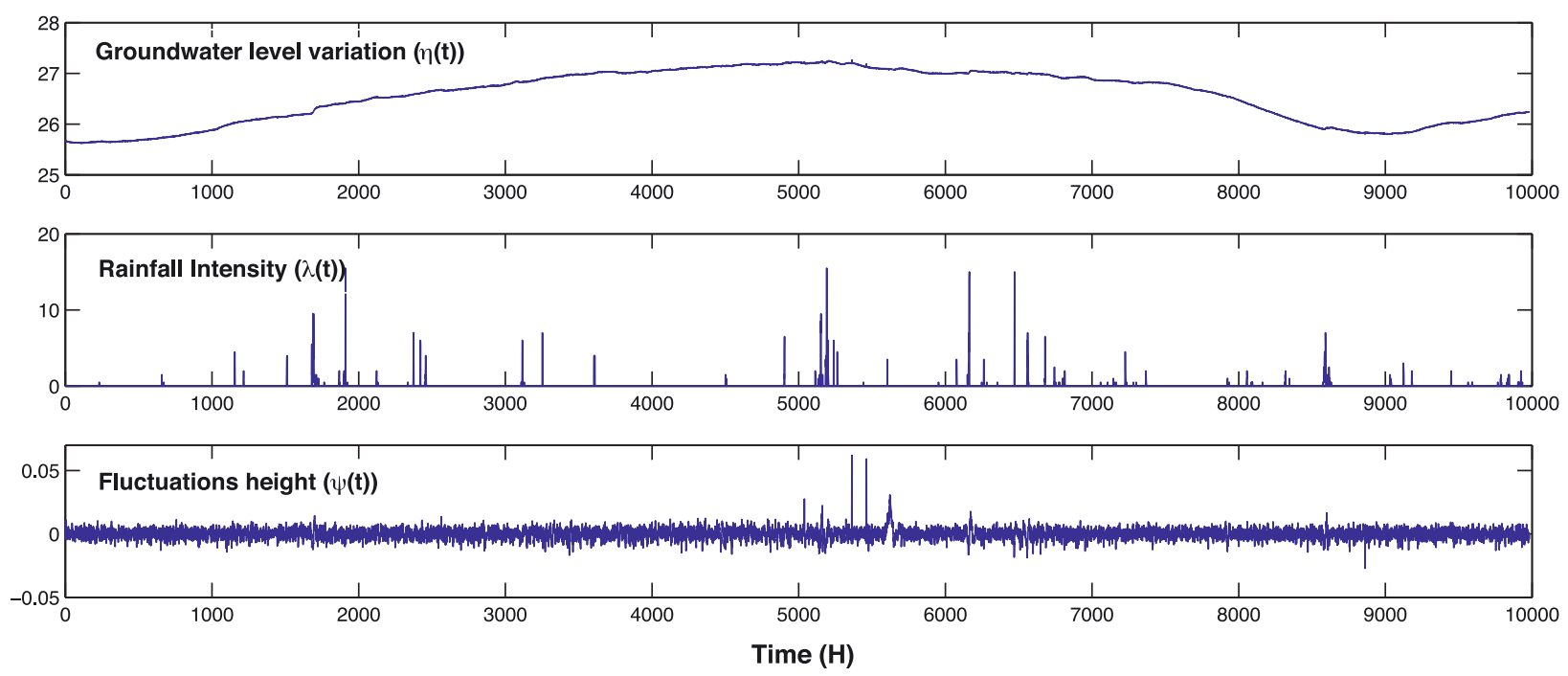

Fig. 3. Top: Groundwater level variation is shown (measured (in m) following the NGF norm) . Middle part: Rainfall intensity (in mm/h). Bottom part: The groundwater level fluctuations (in $\mathrm{m}$ ).
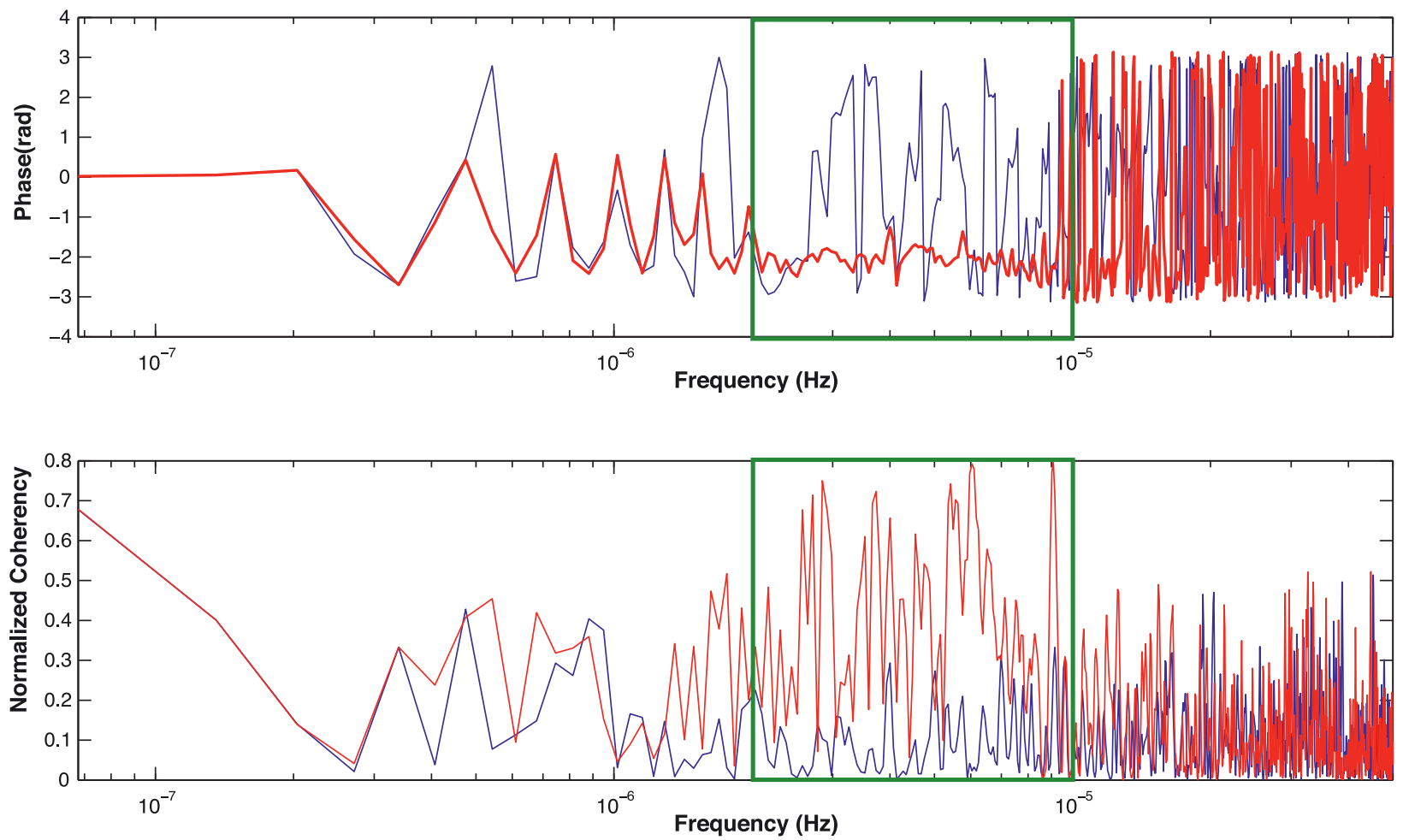

Fig. 4. Normalized coherency function and phase shift between rainfall and groundwater level variations. (For interpretation of the references to color in this figure legend, the reader is referred to the web version of this article.)

formulations given in (8) and (9). The upper part of Fig. 4 represents the phase shift between both time series and the bottom part shows the normalized coherency function. From the coherency function, despite the noisy character of the curve, a coherent structure appears for a frequency domain from $f_{l}=2 e^{-6} \mathrm{~Hz}$ to $f_{h}=1 e^{-5} \mathrm{~Hz}$. The phase information for the same frequency domain presents also a fairly stable evolu- tion that may be seen as a phase signature of the correlation between both time series.

In contrast with these observations, correlation with the surrogate data (blue color in Fig. 4) shows an erratic phase behavior and a considerably low level of coherency with the rainfall time series at the same frequency domain. At frequency values lower than $f_{l}$, real and surrogate data have 

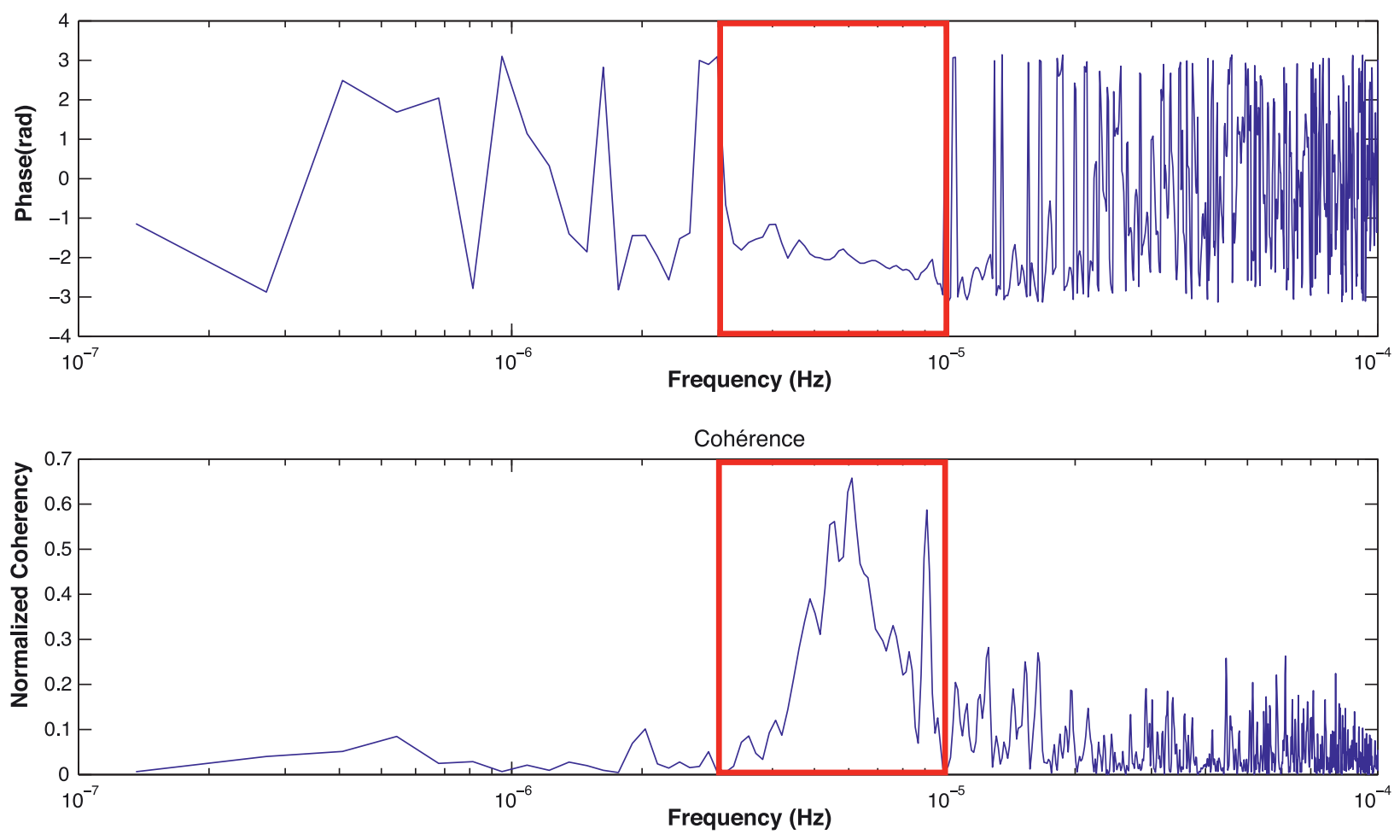

Fig. 5. Normalized coherency function and phase shift between rainfall and groundwater level fluctuations $\psi(t)$.

sensibly comparable behaviors. This indicates that low frequency component of groundwater level does not correlate with rainfall time series and constitutes groundwater annual cycle rather than its response to the rainfall forcing. The same observation may be found for frequency values higher than $f_{h}$. However this later case is less easy to interpret given the lack of precision at high frequency values due to the limitation induced by the frequency sampling value.

These observations and the comparison with the surrogate data results would indicate that the component of the groundwater level variation correlated with rainfall is located at frequency values higher than $f_{l}$. Consequently, to extract the component of the groundwater level variations $\eta(t)$ that is correlated to rainfall time series, we use a digital high-pass filter. The result of the high-pass filtering of the groundwater level variations $\eta(t)$ is a new time series of fluctuations $\psi(t)$. A special care is taken on the filtering process to avoid phase shift between the initial time series $\eta(t)$ and the fluctuations component $\psi(t)$. For such purpose, the high-pass filter was built with the Digital Filter block [33] implemented in the Signal Processing Toolbox of Matlab. A digital filter is characterized by its transfer function that is designed by three main parameters namely, the type of the filter, the order of the filter and the filter function. For the first parameter, one can choose between Infinite Impulse Response (IIR) or Finite Impulse Response (FIR). The primary advantage of IIR filters over FIR filters is that they typically meet a given set of specifications with a much lower filter order than a corresponding FIR. Other interest of the IIR filter is that it allows zero-phase filtering approach (via the Matlab filtfilt function), which eliminates the nonlinear phase dis- tortion. Among the various mathematical filter function, we choose the Butterworth function because it allows to get signal processing filter designed to have as flat a frequency response as possible. It is also referred to as a maximally flat magnitude filter. After some attempts, a fourth order IIR Butterworth high pass frequency with a cutoff frequency at $f_{l}$ is found to be fully satisfactory.

The time plot of the rainfall correlated fluctuations is shown in Fig. 3. In order to complete the verification, we compute now the correlation between the groundwater level fluctuations $\psi(t)$ and the rainfall time series $\lambda(t)$. The results are given in Fig. 5 and show the existence of characteristic peak at frequency $f_{0}=5 e^{-6} \mathrm{~Hz}$. Such peak traduces the existence of statistical time scale $\tau_{0}$ of 2.3 days in the groundwater response to rainfall forcing.

\subsection{Groundwater fluctuations analysis}

\section{Frequency spectrum analysis}

The frequency power spectrum of groundwater fluctuations $\psi(t)$ is plotted in Fig. 6 in double logarithmic coordinates. The spectrum is calculated for a frequency range varying from $1 e^{-6} \mathrm{~Hz}$ up to the Nyquist frequency $f_{N}=\frac{1}{2 \Delta t}$ which is equal to $5 e^{-4} \mathrm{~Hz}$. A quasi continuous power spectrum embedded in noise can be found especially at high frequency. The power spectrum shows a broad maximum around a frequency peak at $f=6.5 e^{-6} \mathrm{~Hz}$. As a general remark, the broadness of the spectrum peak and the continuous aspect of this later are rather indicative of stochastic dynamic than a deterministic one. This would be confirmed later by the higher order analysis that provides a quantification of the 


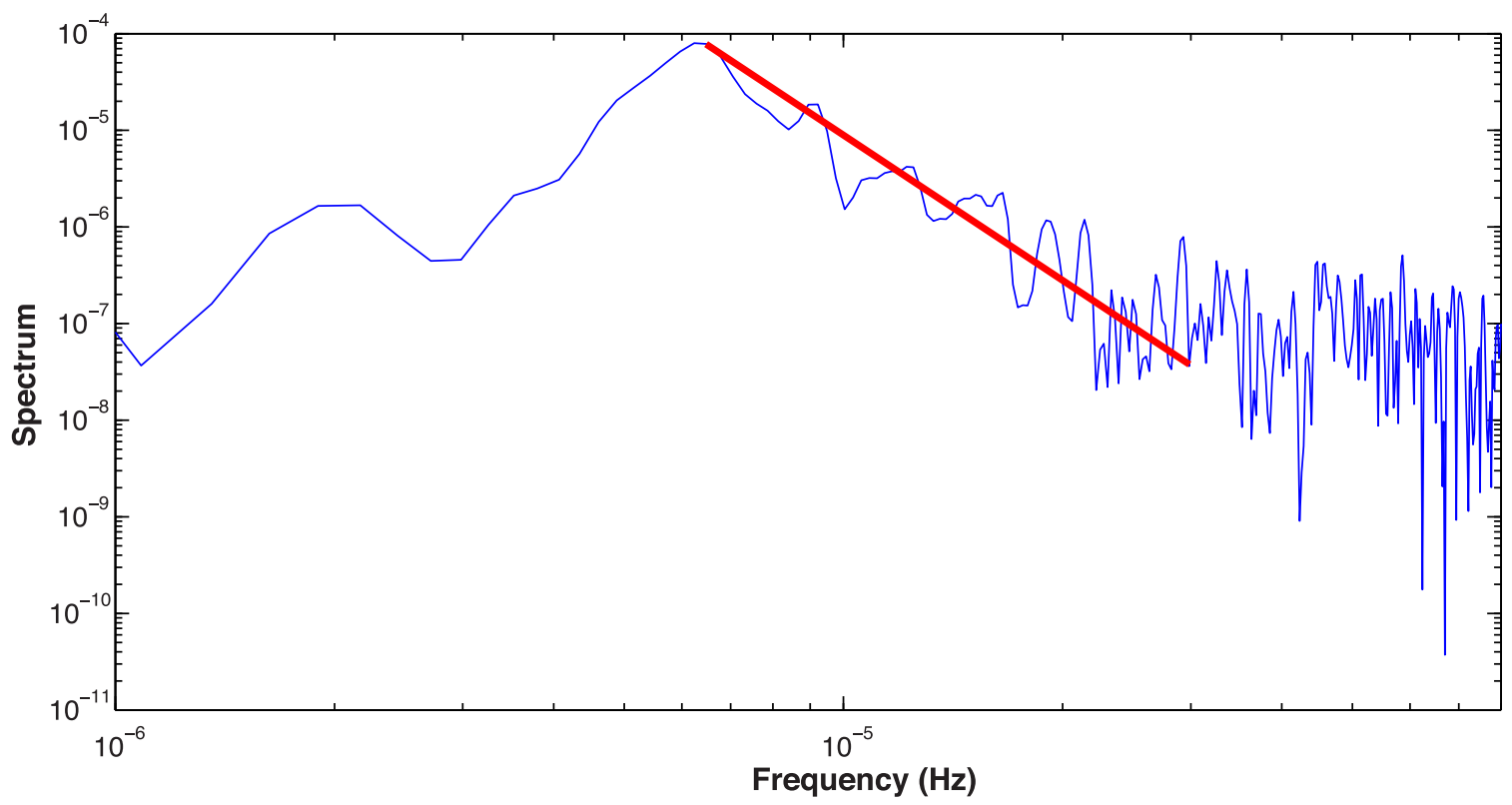

Fig. 6. Frequency spectrum of groundwater level fluctuations $\psi(t)$.

nonlinear interactions between Fourier components. Such nonlinear interactions are known to be a necessary condition to low dimensional chaotic dynamics. Looking at the whole power spectrum it appears that this later exhibits a region of linear trend for a frequency range from $6.5 e^{-6} \mathrm{~Hz}$ up to $3 e^{-5} \mathrm{~Hz}$. This trend can be described by a power law regression as:

$S_{\psi}(f)=A f^{-\kappa}$

Value of the parameter $\kappa$ in Eq. (16) are found to be equal to 0.602 . The frequency domain of power law is included into the correlated interval with the rainfall forcing. This seems to show the existence of a fractal power law property of the correlated rainfall component of the groundwater level fluctuations. However, we do not have found explanation for the departure from the power law observed at the high frequency part of the spectrum.

\section{Higher order spectrum}

In order to attempt to describe the groundwater level fluctuations as a chaotic dynamical system, we compute the bicoherence function. Because of real-valued nature of the data, only the non-redundant domain of the bicoherence function is represented in a normalized axis (see for example [13]). In Figs. 7 and 8, the $x$-axis and $y$-axis represent frequency domain in dimensionless unit by dividing frequencies by $F_{0}$ which is the frequency of spectrum peak. Here, we try to consider the observations in terms of interactions between Fourier components. As shown in Fig. 7, the bicoherence function exhibits high values (up to 0.8 ) in a large part of the frequency domain upper than the frequency peak (at $5.5 e^{-5} \mathrm{~Hz}$ ) without a precise pattern excepted at a low frequency that seems to indicate a phase coupling effect around the main peak frequency. Due to the lack of specific patterns which are the hallmark of phase relationships, the present result does not allow us to describe the groundwater level fluctuations dynamic as a chaotic deterministic sys- tem induced by nonlinear quadratic interactions. Nevertheless, the high values of the bicoherence reflect the presence of more complex nonlinear interactions involving a large number of Fourier components. Here, we note that this result differs from those obtained by Wang and Peng [44]in which a low dimensional deterministic chaos is found to govern the groundwater level dynamics at large time scale (they studied a time series of 25 years with time resolution of five days).

Despite this fact, comparison with the surrogate data results gives us more useful information. Indeed, in contrast to the real data results, the surrogate data has a zero value of the bicoherence function (see Fig. 8). This is not also surprising with regard to the surrogate construction principle that is based on phase randomization. As a consequence, the high values of the bicoherence of the real data are undoubtely linked to its phase structure. Owing to the fact that higher order statistics of the real data does not vanish, thus the real data are probably of non-Gaussian nature. At the present step of our analysis, we assume that the groundwater level fluctuations $\psi(t)$ have fractal and non-Gaussian nature (see for example in [40]). This later property will be more investigated in the next section.

\section{Probability distribution}

In this section, we estimate the probability distribution function of the groundwater level fluctuations. The probability distribution function of $\psi(t)$ is depicted in double linear and in double logarithmic coordinates in the Fig. 10. The data probability density is represented with the symbol 'o' in the figure. Probability distribution models are drawn with solid and dashed lines. As a first comment, the random like character of the fluctuations is attested by a density probability that is close to Gaussian law in its middle part while the asymptotic behavior of probability distribution shows heavy tails properties that are well fitted with an alpha stable probability distribution. By fitting the data probability distribution function, we find a stability index of $\alpha=1.90$ that is 


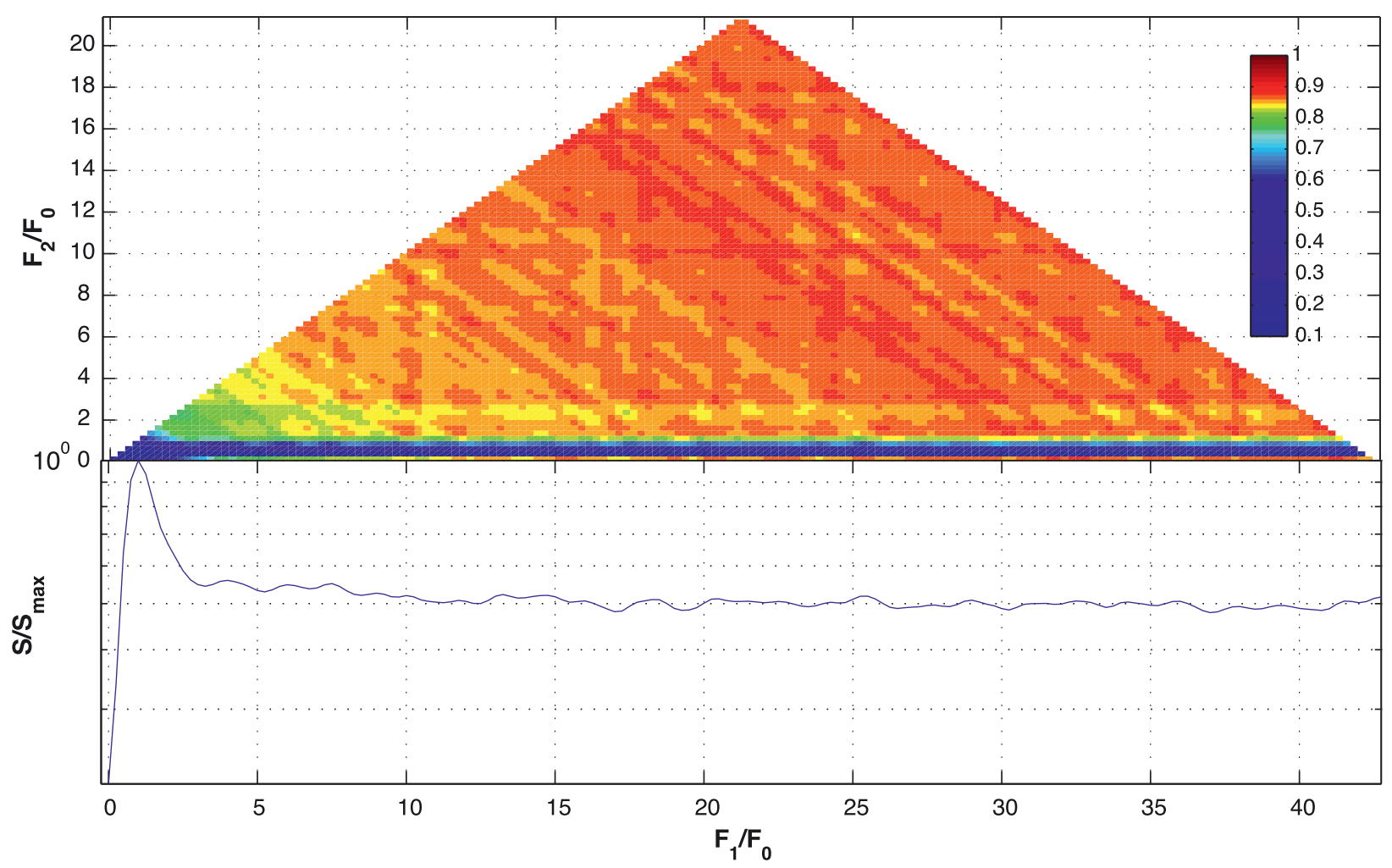

Fig. 7. Top: Bicoherency Function of the groundwater level fluctuations $\psi(t)$. Bottom: Spectrum function in linear scale.

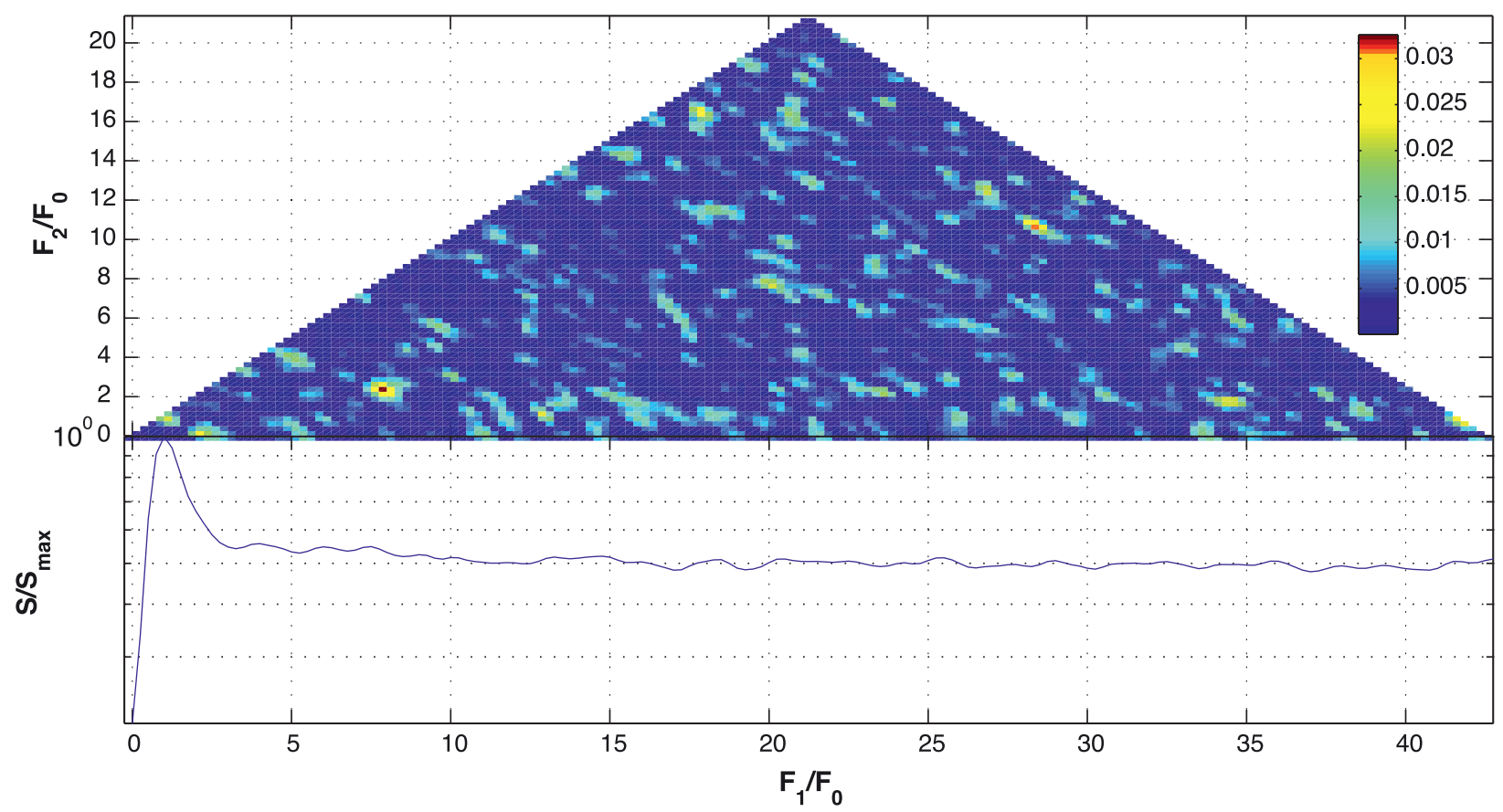

Fig. 8. Top: Bicoherency Function of the surrogate of the groundwater level fluctuations $\psi(t)$. Bottom: Spectrum function in linear scale. 
indicative of a non-Gaussian character. A negative weak value of the skewness parameter $\beta=-0.36$ is also found traducing an asymmetry property on the fluctuations. The scale parameter is found to be equal to $\sigma=0.6$ that is a well comparable value with the normalized Gaussian standard devia$\operatorname{tion}\left(\sim S_{2}(0,1 / \sqrt{2}, 0)\right)$.

\section{Estimating long range memory with Hurst exponent}

Another way to state the random nature of the physical process is to analyze its long term behavior. In other words, we will test if the time series $\psi$ exhibits long term memory or long range dependence. Long range dependence means that the physical process depicted by the data has an underlying trend with some degree of autocorrelation. Such property is known to be related to self-affine characteristic and fractal nature of the data. Indeed, self-affine signals may be described by a finite fractal dimension which is related to the Hurst exponent. It means also that in this case, the process is embedded in a low dimensional phase space. This lets the opportunity to model the process by nonlinear chaotic deterministic laws with only few parameters . However, we note that the existence of long range dependence does not provide an ability to discriminate between stochastic or deterministic nature for the studied process. Another important property related to the self-affinity is the scale invariance. This later is vital in the sense that with scale invariance property, short length data may get insight on more large scale behavior. As a consequence, long range dependence was widely studied for many years in hydrology and geophysics. In this paper, we will estimate the Hurst exponent that is the oldest and the best-known among the various estimators of the intensity of long range dependence proposed in the literature. The Hurst exponent may be defined as follows: a stochastic process $\Psi(t)$ has a Hurst exponent $H$ if it verifies:

$\Psi(c t) \stackrel{d}{=} c^{H} \Psi(t) \quad \forall t \geq 0, \forall c>0$

Eq. (17) is written in the sense of finite-dimensional distributions that means for any $d \geq 1$ sequence of time points $t_{1}, \ldots, t_{d}$ and any positive constant c, $e^{-H}\left(\Psi\left(t_{1}\right), \ldots, \Psi\left(t_{d}\right)\right)$ has the same distribution as $\left(\Psi\left(c t_{1}\right), \ldots, \Psi\left(c t_{d}\right)\right)$.

A wide range of techniques are now available to estimate the Hurst exponent $\mathrm{H}$. The Hurst exponent is normalized between the values 0 and 1. From (17) it is easy to check that for example Brownian motion that is memoryless process has self-similarity with $H=0.5$. Thus, values other $H=0.5$ denote the presence of memory effect. Particular interest focuses on the hypothesis that $H>0.5$, indicating relatively long-range dependence that implies a persistent time series characterized by long term memory effects.

The traditional method to estimate this exponent is the rescaled adjusted range analysis ( $R / S$ analysis), that has its roots in early work of the British hydrologist Hurst [12], who investigated dependence properties of phenomena such as levels of the River Nile. Of interest is that R/S analysis is nonparametric, meaning there is no assumption or requirement of the shape of the underlying distribution. Then, the result provides compatible information with the probability distribution analysis made above and may be interpreted in a consistent way with this later. Here we use the fast algorithm developed in [1]. Details on R/S analysis presentation is now available in a large number of publications (see e.g. [1,26]). A

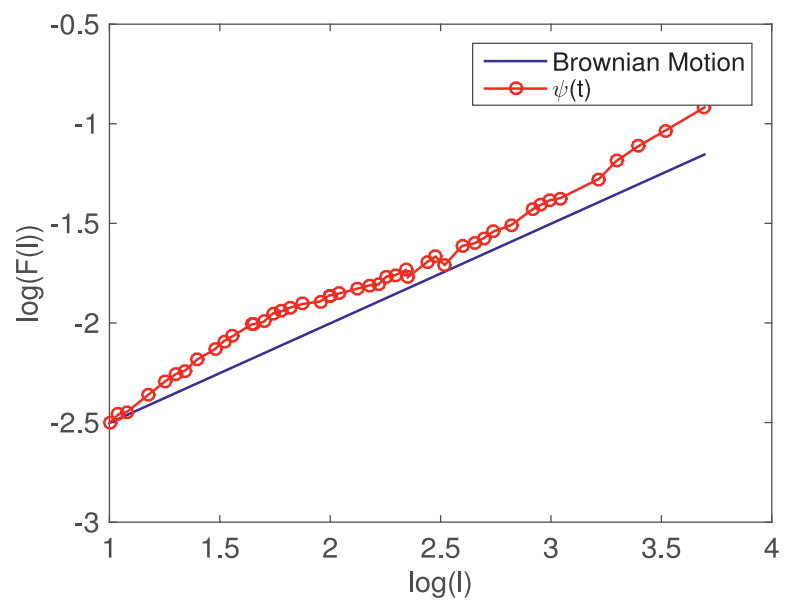

Fig. 9. Detrended Fluctuations analysis of the time series $\psi(t)$.

value of the Hurst exponent $H=0.54$ is found for the groundwater fluctuations time series $\psi(t)$.

In order to confirm the result of the R/S analysis, we will use also the Detrended Fluctuation Analysis (DFA). The DFA was first proposed by Peng et al. [34,35] to quantify scaling properties of biological processes. The method can be summarized as follows [36]. From the original time series $\left\{\psi\left(t_{1}\right), \psi\left(t_{2}\right), \ldots, \psi\left(t_{i}\right), \ldots, \psi\left(t_{n}\right)\right\}$ we note $y\left(t_{j}\right)$ a cumulative time series defined as:

$y(j)=\sum_{i=1}^{j}\left[\psi\left(t_{i}\right)-<\psi>\right]$

where $\langle\psi\rangle$ is the average value of the time series $\psi\left(t_{j}\right)$. Then the cumulative time series is divided into $N_{l}=(n / l)$ non-overlapping segments of length $l$. For each segment $v=$ $1,2, \ldots, N_{l}$, a fit by least-squares line is made giving a new set of time series noted as $\tilde{Y}_{\nu}$. Then, we calculate the root mean square of the cumulative and the detrended time series.

$F(v, l)=\sqrt{\frac{1}{l} \sum_{j=1}^{l}\left[Y((v-1) l+j)-\tilde{Y}_{\nu}(j)\right]^{2}}$

Finally, the mean values of $F(v, l)$ are calculated as follows

$F(l)=\frac{1}{N_{l}} \sum_{\nu=1}^{N_{l}} F(\nu, l)$

By plotting $\log (F(l))$ against $\log (l)$, an almost linear relationship may be found that indicates the presence of a power-law scaling of the form $\mathrm{Cl}^{\mathrm{H}}$ where $\mathrm{C}$ is a constant. The result from the groundwater fluctuations $\psi(t)$ is given in Fig. 9. The estimated value of the Hurst exponent with DFA method is found to be $H=0.5343$. The estimation was performed using three confidence levels as shown in Table 3.

We note that detailed aspects on the construction of the confidence intervals may be found in the paper of Weron [45]. Of interest is that the value of the Hurst exponent given by the $\mathrm{R} / \mathrm{S}$ analysis is inside the confidence intervals for all three levels. Such result suggests that the value of the Hurst exponent from the R/S analysis method provide an acceptable estimate of the exact one. 
a

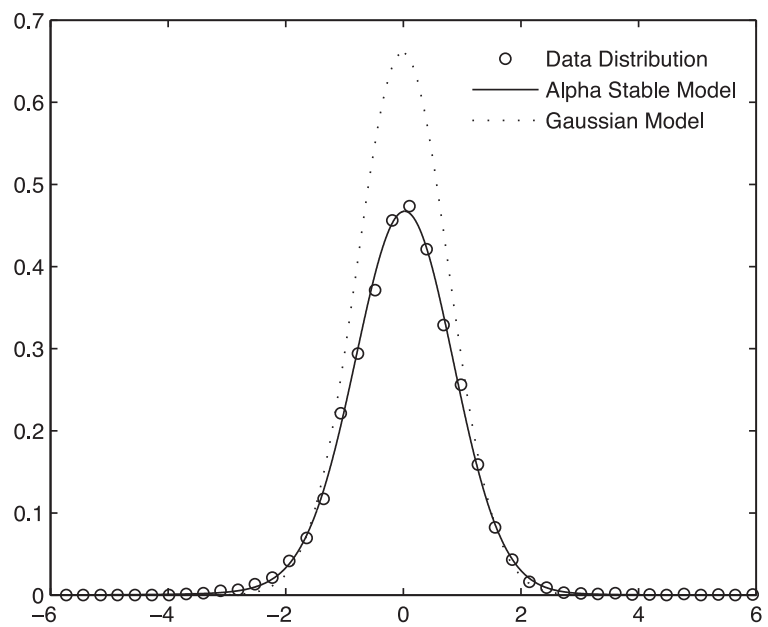

b

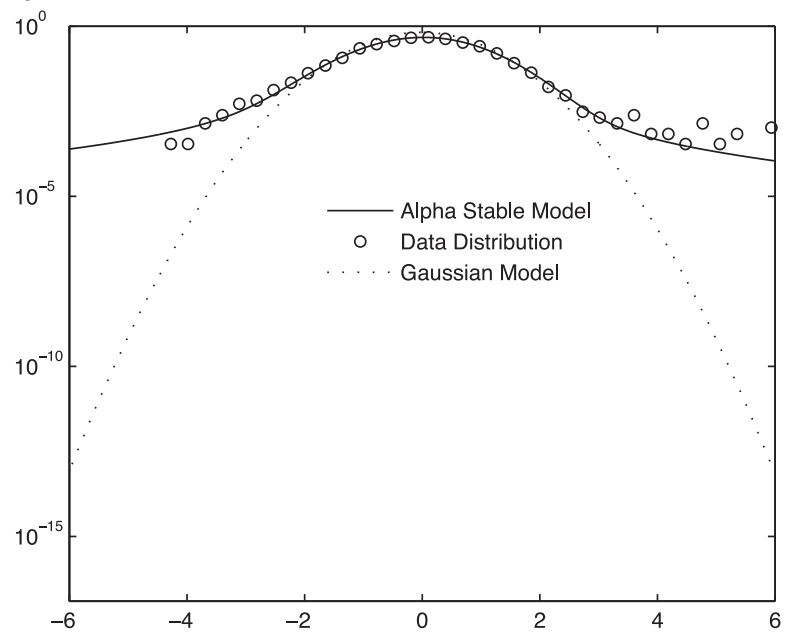

Fig. 10. Probability distribution function of groundwater level fluctuations $\psi(t)$ in linear (left) and logarithmic (right) coordinates. The stability index of the stable law is equal to $\alpha=1.9$.

Table 3

Two-sided empirical confidence intervals for the Hurst exponent estimation from the DFA method.

\begin{tabular}{lll}
\hline Conf. Low & Conf. High & Level \\
\hline 0.4469 & 0.5416 & 0.9000 \\
0.4376 & 0.5507 & 0.9500 \\
0.4186 & 0.5662 & 0.9900 \\
\hline
\end{tabular}

\section{index \\ Relationship between Hurst exponent and stability}

To model stochastic processes with long range correlation, generalization of the Brownian motion with the fractional Brownian motion $(H>0.5)$ was carried out by Mandelbrot [25]. However, these processes are Gaussian have finite variance and then are not suitable to represent physical process with infinite variance laws such as the alpha stable distribution. According to the theorem of Taqqu [42], for infinite variance process, a relationship exists between the stability index and the Hurst exponent:

$H=(3-\alpha) / 2$

A striking fact is that the estimated value of the stability index from data matches Eq. (21). The departure between the value of stability index estimated from real data and that deducted from Taqqu theorem by the relation (21) is found of order of one percent. Also, our Hurst exponent value is found to be exactly the same as that obtained by [46] and [47].

To resume the analysis, it is found that groundwater level fluctuations $\psi(t)$ is of fractal nature with non-Gaussian heavy tail probability distribution marked by a persistent behavior. Significations and implications of these results will be addressed in the last section of this paper.

\section{Conclusions and discussions}

The main purpose of the present paper was to characterize the dynamical behavior of groundwater level fluctuations due to the rainfall process. The study deals with the case of groundwater located in the South-East region of France in which it is known that the rainfall process is marked by an accentuated occurrences of fast events. In the first step of the paper, the component correlated with rainfall is extracted from the global level fluctuations of the groundwater. By applying spectral analysis, the energy spectrum of this component of the groundwater level fluctuations is found to have an invariant scaling law. Indeed, a power law is found at a frequency domain corresponding to the frequency interval correlation with rainfall time series. This result would indicate a fractal nature of the level fluctuations. It is also consistent with the results found recently in the study of $[46,47]$. However, the high frequency part of the spectrum does not follow the power law. We do not have exact explanation about this behavior that is reminiscent of noise effect. However, as pointed out by Serletis [38], noise does not affect the fractal structure of the time series. To access on more complete information, higher order spectral analysis is carried out in order to attempt to describe the underlying dynamics as a low dimensional chaotic system dominated by nonlinear interactions between Fourier components. Despite the high values of the corresponding bicoherence function, it does not allow us to describe the dynamic of the groundwater level fluctuations as the effects of nonlinear interactions between few Fourier components. However comparison between real and surrogate data shows that the real data time series has non-vanishing higher order moments linked to its phase structure that traduces a non-Gaussian character. According to this constatation, the probability distribution of the groundwater level fluctuations is investigated. As shown in Fig. 10 heavy tailed probability distribution is found from the data time series. Also, the Lévy stable laws fit well the probability distribution of the data. A stability index of order $\alpha=1.9$ is found revealing a non-Gaussian behavior of the groundwater level fluctuations. The probability distribution is slightly skewed. This result is reminiscent of earlier works such as in [3]. On the other hand, the Hurst exponent was 
estimated from the data and found to be around of 0.54 . Here also, our result corroborates the Hurst exponent value found in $[46,47]$. This value is indicative of a persistent behavior that is compatible with fractal property revealed by the spectrum analysis. From more theoretical consideration, our values check almost fairly the relationship between the stability index of the probability distribution function and the Hurst exponent as established by the mathematical theorem in [42]. The combination of the heavy tailed probability distribution, the fractal behavior of the energy distribution found in the spectral analysis and the persistent behavior constitutes a complete proof on the existence of long memory effects inside the groundwater fluctuation dynamics. Our results are comparable to these found by Peng et al. [35] concerning a biological system. With regard to the modeling aspect, our results raise a question on the validity of Brownian or fractional Brownian motions (see for examples in $[4,22,47]$ ) to model the groundwater recharge dynamic known that these motions belong to the class of Gaussian finite variance processes (see for example [29]). Also, the characteristics of this long-memory process is essential to forecasting problem [5] and have to be taken into account in models. A possible way to reconcile these two constraints would be the appeal to the class of fractional Lévy motions (see for example [24]). Fractional Lévy motions are based on the Lévy stable laws and may be declined as a random walk that generates anomalous diffusion (see for example [2]).

According to general theory of the subsurface hydrology, the present results have consequences on the others quantities that define the groundwater system. In particular, following the transfer function models established by Gelhar [9], the fractal non-Gaussian properties found here may concern also the dynamics of the groundwater recharge.

More generally, the implications of these results to the characterization of mass transfer process in the heterogeneous media that is the soil layer may be essential. One specific question that we have raised at the origin of this paper was the description of the transfer of the mass of water from natural rainfall events inside the soil layer. For this purpose, it is straightforward that no direct reliable method is available to study mass transfer process through the soil layer under in situ condition. To address this goal, the groundwater level fluctuations and the rainfall events were observed in a synchronized manner. In other words, instead of studying the soil layer as a system, we have analyzed its input and output. The fractal non-Gaussian character with long memory behavior found in the groundwater level fluctuations and on the other hand, the presence of the same properties recognized in the rainfall process (see for example [23,32,37]) suggest that the transport process inside the soil may be also of non-Gaussian nature with a memory effect. Such idea involves modeling of the the mass spread as an anomalous diffusion law that is based on non-local operator (see for examples $[20,28,30])$.

To end this paper, it is important to recall that the results given herein are limited in some aspects of the temporal evolution of the groundwater level fluctuations at the given locations. This study deals with the context of small scale space for which the effect of spatial variability was found negligible. However, at more large scale, lateral input and the base flow effects would be important and have to be taken in account by a time-space analysis.

\section{Acknowledgments}

The authors would like to thank F. Tison for valuable technical supports. We are also grateful to E. Michel for helpful discussions on the draft of the paper and for his help in improving the text.

\section{References}

[1] Blok HJ. On the nature of the stock market: Simulations and experiments Phd thesis. University of British Columbia; 2000.

[2] Bellazzini J. Random walks and coupling in complex systems. Chaos Solitons Fract 2004. doi:10.1016/S0960-0779(03)00439-9.

[3] Benson D, Wheatcraft S, Meerschaert M. Application of a fractional advection-dispersion equation. Water Resour Res 2000;36(6):1403-12.

[4] Bierkens MFP. Modeling water table fluctuations by means of a stochastic differential equation. Water Resour Res 1998;34(10):248599.

[5] Butterworth JA, Schulze RE, Simmonds LP, Moriarty P, Mugabe F. Hydrological processes and water resources management in a dryland environnement IV: long-term groundwater level fluctuations due to variation in rainfall. Hydrol Earth Syst Sci 1999;3(3):353-61.

[6] Dickinson JE, Hanson RT, Ferré TPA, Leake SA. Inferring time-varying recharge from inverse analysis of long-term water levels. Water Res Resour 2004;40:W07403. doi:10.1029/2003WR002650.

[7] Liliana DP, Doussan C, Ruy S, Gaudu JC, Pallut R, Renard D. Site Ateliers "Fontanile", Infiltration, recharges, écoulements préférentiels et transport de polluants vers la nappe. Technical report. INRA; 2004

[8] Finke PA, Brus DJ, Bierkens MFP, Hoogland T, Knotters M, de Vries F. Mapping groundwater dynamics using multiple sources of exhaustive high resolution data. Geoderma 2004:123(1-2):23-39.

[9] Gehlar LW. Stochastic subsurface hydrology. From theory to applications. Water Resour Res 1986;22(9):135-45.

[10] Gnedenko BV, Kolmogorov AN. Limit distributions for sums of independent random variables. Bull Amer Math Soc 1956;62(1):50-2.

[11] Hinich MJ. Testing for Gaussianity and linearity of a stationary time series. J Time Ser Anal 1982;3(3):169-76.

[12] Hurst HE. The long-term storage capacity of reservoirs. Trans Amer Soc Civil Eng 1951;116:770-99.

[13] Joelson M, Dudok Dewit T, Dussouliez P, Ramamonjiarisoa A. Searching for chaotic and deterministic features in laboratory surface waves Nonlinear Process Geophys 2000;7:37-48.

[14] Joelson M, Néel MC. On alpha stable distribution of wind driven water surface wave slope. Chaos 2008;18:033117. http://dx.doi.org/10.1063/1. 2955742.

[15] Kim YC, Powers EJ. Digital bispectral analysis and its applications to nonlinear wave analysis. IEEE Trans Plasma Sci, PS 1979;7(120):120_ 31.

[16] Kogon SM, Williams DB. Characteristic function based estimation of stable parameters. In: Adler R, Feldman R, Taqqu M, editors. A practical guide to heavy tails. Boston: Birkhauser; 1998.

[17] Koopmans LH. The spectral analysis of time series. New York and London: Academic Press; 1970. p. 366.ISBN 0-12-419250-5

[18] Koutrouvelis IA. Regression-type estimation of the parameters of stable laws. J Am Stat Assoc 1980;75:918-28.

[19] LeroyM.. Estimation de l'incertitude de mesure des précipitations. 2000. Note Interne DSO, N42, Météo-France.

[20] Levy M, Berkowitz B. Measurement and analysis of non-Fickian dispersion in heterogeneous porous media. J Contam Hydrol 2003. doi:10. 1016/S0169-7722(02)00204-8.

[21] Lévy P. Calcul des probabilités. Paris: Gauthier Villars; 1925

[22] Li Z, Zhang Y-K. Quantifying fractal dynamics of groundwater systems with detrended fluctuation analysis. J Hydrol 2007;336(1-2):139-46.

[23] Lovejoy S, Mandelbrot B. Fractal properties of rain, and a fractal model. Ser A Tellus 1985;37:209-32.

[24] Lowen SB, Teich MC. Power law shot noise. IEEE Trans Inf Theory 1990;36(6):1302-18.

[25] Mandelbrot BB, Van Ness JW. Fractional Brownian motions, fractional noises and applications. SIAM Rev 1968;10(4):422-37.

[26] Mandelbrot BB, Wallis JR. Some long-run properties of geophysical records. Water Resour Res 1969;5(2):321?340.

[27] Mendel JM. STutorial on higher-order statistics (spectra) in signal processing and system theory: theoretical results and some applications. Proc IEEE 1991;79(3):278-305. 
[28] Metzler R, Chechkinb AV, Goncharb VY, Klafter J. Some fundamental aspects of Lévy flights. Chaos Solitons Fract 2007. doi:10.1016/j.chaos. 2007.01.055.

[29] Molz FJ, Rajaram H, Lu S. Stochastic fractal-based models of heterogeneity in subsurface hydrology: Origins, applications, and future research questions. Rev Geophys 2004;42(1). doi:10.1029/ 2003RG000126.

[30] Néel MC, Abdennadher A, Joelson M. Fractional Fick's law: the direct way. J Phys A: Math Theor 2007;40. doi:10.1088/1751-8113/40/29/007.

[31] Nikias C, Petropulu AP. Higher order spectra analysis: a non-linear signal processing framework.. Prentice Hall; 1993. p. 528. ISBN-13: 9780136782100.

[32] Olsson J. Limits and characteristics of the multifractal behavior of a high resolution rainfall time-series. Nonlinear Process Geophys 1995;2:239.

[33] Oppenheim AV, Schafer RW. Discrete-time signal processing. PrenticeHall; 1989. p. 311-12.

[34] Peng C-K, Buldyrev SV, Havtin S, Simons M, Stanley HE, Goldberger AL. Mosaic organization of DNA nucleotides. Phys Rev E 1994;49(2):16859.

[35] Peng C-K, Mietus J, Hausdorff JM, Havtin S, Stanley HE, Goldberger AL. Long-range anti-correlation and non-Gaussian behavior of the Heart Beat. Phys Rev Lett 1993;70(9):1685-9.

[36] Rakhshandehroo GR, Amiri SM. Evaluating fractal behavior in groundwater level fluctuations time series. J Hydrol 2012. doi:10.1016/j. jhydrol.2012.07.030.

[37] Rehman S. Study of Saudi Arabian climatic conditions using Hurst exponent and climatic predictability index. Chaos Solitons Fract 2007. doi:10.1016/j.chaos.2007.01.079.

[38] Serletis D. Effect of noise on fractal structure. Chaos Solitons Fract 2007. doi:10.106/j.chaos.2007.01.031.
[39] Shang P, Kamae S. Fractal nature of time series in the sediment transport phenomenon. 2005. doi: 10.1016/j.chaos.2005.01.051.

[40] Silva LBM, Vermelho MVD, Lyra ML, Viswanathan GM. Multifractal detrended fluctuation analysis of analog random multiplicative processes. Chaos Solitons Fract 2008. doi:10.1016/j.chaos.2008.10.027.

[41] Tankersley CD, Graham WD, Hatfield K. Comparison of univariate and transfer function models of groundwater fluctuations. Water Resour Res 1993;29(10):3517-33.

[42] Taqqu MS, Willinger W, Sherman R. Proof of fundamental result in selfsimilar traffic modelling. Comput Commun Rev 1997;27:5-23.

[43] Theiler J, Eubank S, Longlin A, Galdikrian B, Farmer JD. Testing for nonlinearity in time series: the method of surrogate data. Phys D 1992;58:77-94

[44] WanG W, Peng S. Chaotic analysis on series of groundwater depth in hetao irrigation district. Proc ISKE 2007. doi:10.2991/iske.2007.161.

[45] Weron R. Estimating long-range dependence: finite sample properties and confidence intervals. Phys A 2002;312(12):285-99. doi:10.1016/ S0378-4371(02)00961-5.

[46] Zhang Y-K, Schilling K. Temporal scaling of hydraulic head and river base flow and its implication for groundwater recharge. Water Resour Res 2004;40:W03504. doi:10.1029/2003WR002094.

[47] Zhang Y-K, Li Z. Temporal scaling of hydraulic head fluctuations: nonstationary spectral analyses and numerical simulations. Water Resour Res 2005;41:W07031. doi:10.1029/2004WR003797.

[48] Zhongwei L, Zhang Y-K. Quantifying fractal dynamics of groundwater systems with detrended fluctuations analysis. J Hydrol 2007. doi:10. 1016/j.jhydrol.2006.12.017.

[49] Zolotarev MV. One dimensional stable distributions. Am Math Soc 1986;65:284. Translation of the original 1983 Russian. 